\title{
Optimal placement of electrodes for treatment of post-stroke dysphagia by neuromuscular electrical stimulation combined with effortful swallowing
}

\author{
Jae-Won $\underline{\text { Huh }}^{1,2}$, MD, Eunhee $\underline{\text { Park }}^{1,3}$, MD, PhD, Yu-Sun $\underline{M i n}^{1,3}$, MD, Ae Ryoung $\underline{K i m}^{1,2}$, MD, Won-Jong $\underline{Y a n g}^{1,3}$, MD,
} Hyun-Min $\underline{\mathrm{Oh}}^{1,2}$, MD, Tae-Woo ${\underline{\mathrm{Nam}^{1,3}}}^{1,} \mathrm{MD}, \mathrm{Tae}-\mathrm{Du} \underline{\mathrm{Jung}}^{1,3}$, MD, PhD

INTRODUCTION We aimed to determine the optimal placement of electrodes for neuromuscular electrical stimulation (NMES) for post-stroke dysphagia therapy.

METHODS 31 patients with post-stroke dysphagia were randomised to three groups according to NMES electrode placement. In Group A ( $n=10)$, two pairs of electrodes were attached horizontally on the suprahyoid and infrahyoid muscles. In Group B ( $n=11)$, one pair of electrodes was attached horizontally on the suprahyoid muscles while the other was attached vertically on the infrahyoid muscles. In Group C $(n=10)$, the electrodes were attached vertically, with one pair above the hyoid bone and the other above the cricoid cartilage. All patients received rehabilitation treatment via NMES combined with effortful swallowing training five times weekly for four weeks. The effect of NMES electrode placement was assessed in terms of the Functional Dysphagia Scale (FDS) and Dysphagia Outcome and Severity Scale (DOSS) scores. RESULTS Group A showed significantly greater improvement than Group B in overall FDS $(p=0.009)$ and pharyngealphase FDS (FDS-P; $p=0.005$ ) scores. Group A also showed significant improvement when compared with Group $C$ in overall FDS $(p=0.001)$ and FDS-P $(p=0.001)$ scores.

CONCLUSION Horizontal placement of the NMES electrodes on the suprahyoid and infrahyoid muscles for the treatment of post-stroke dysphagia by NMES combined with effortful swallowing was more effective than the horizontal and vertical placement of electrodes on the suprahyoid and infrahyoid muscles, respectively, and their vertical placement above the hyoid bone and cricoid cartilage.

Keywords: deglutition disorder, dysphagia, electrical stimulation, stroke

\section{INTRODUCTION}

Swallowing dysfunction, or dysphagia, is known to occur in up to $76 \%$ of stroke patients. Patients with post-stroke dysphagia develop complications, such as malnutrition, dehydration and pneumonia, which can delay functional recovery. Pneumonia accounts for at least $10 \%$ of post-stroke deaths within 30 days of hospitalisation for stroke. ${ }^{(1-4)}$ Therefore, early detection and treatment of dysphagia is crucial for recovery.

Conventional dysphagia treatment consists of oropharyngeal exercises, compensation manoeuvres and oropharyngeal stimulation. ${ }^{(5)}$ Singh and Hamdy recommended that standard treatment for post-stroke dysphagia includes compensatory manoeuvres, such as postural adjustment, Mendelsohn manoeuvre, supraglottic swallowing and effortful swallowing. ${ }^{(6)}$ Among these, effortful swallowing was shown to significantly decrease the residue in the oral cavity, increase oral pressure and improve hyoid bone elevation during swallowing in healthy adults. $^{(7)}$

Recent studies have reported that stimulating the pharyngeal muscles using neuromuscular electrical stimulation (NMES) was effective for improving swallowing function. ${ }^{(8-10)}$ By stimulating the mylohyoid, geniohyoid and thyrohyoid muscles, Burnett et al achieved laryngeal elevation in healthy volunteers. ${ }^{\left({ }^{8}\right)}$ Leelamanit et al reported that synchronised electrical stimulation of the thyrohyoid muscle during swallowing has a therapeutic effect in patients with dysphagia. ${ }^{(9)} \mathrm{A}$ retrospective study of NMES reported that NMES therapy appeared to be beneficial for patients with mild-to-moderate dysphagia. ${ }^{(10)}$

In previous studies on the effect of NMES therapy for poststroke dysphagia, the following three therapeutic methods, according to NMES placement, were most commonly used. ${ }^{(10-16)}$ Firstly, two pairs of electrodes were attached horizontally on the suprahyoid and infrahyoid muscles. ${ }^{(10-12)}$ Secondly, one pair of electrodes was attached horizontally on the suprahyoid muscles while the other was attached vertically on the infrahyoid muscles. ${ }^{(10,13,14)}$ Lastly, two pairs of electrodes were attached vertically above the hyoid bone and cricoid cartilage. ${ }^{(10,15,16)}$ However, there is no clinical consensus on which NMES electrode placement is most effective for dysphagia therapy.

Therapeutic methods that combine NMES with effortful swallowing training have been proven to be beneficial for swallowing function improvement. ${ }^{(17)}$ In the present study, we hypothesised that electrode placement may influence the therapeutic effect of NMES combined with effortful swallowing in patients with post-stroke dysphagia. The study was designed to apply the therapeutic methods that combined NMES with effortful swallowing training with the three most commonly used placements of electrodes. The study objective was to determine

\footnotetext{
Department of Rehabilitation Medicine, School of Medicine, Kyungpook National University, ${ }^{2}$ Department of Rehabilitation Medicine, Kyungpook National University Hospital, ${ }^{3}$ Department of Rehabilitation Medicine, Kyungpook National University Chilgok Hospital, Daegu, South Korea

Correspondence: Dr Tae-Du Jung, Associate Professor, Department of Rehabilitation Medicine, Kyungpook National University Chilgok Hospital, No. 807 Hoguk-ro, Buk-gu, Daegu 41404, South Korea. teeed0522@hanmail.net
} 

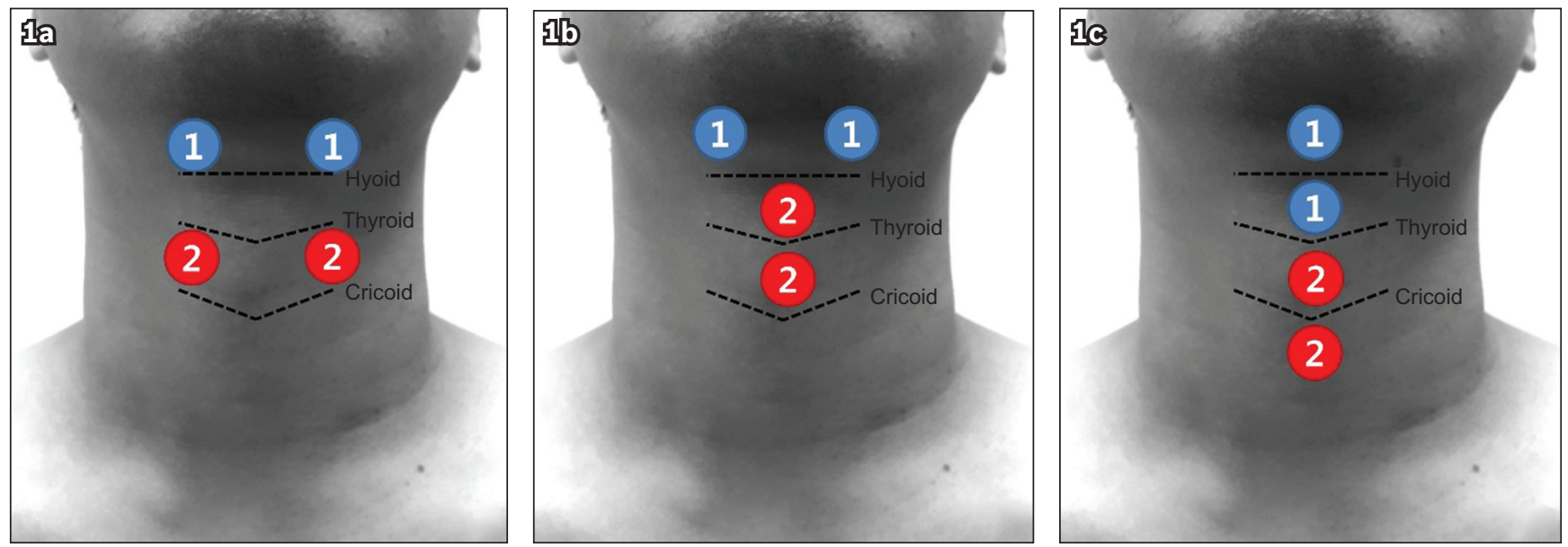

Fig. 1 Image shows the placement of neuromuscular electrical stimulation electrodes, with patients being randomised into three groups based on positioning of electrodes on the muscles above and below the hyoid bone, (a) Group A, (b) Group B and (c) Group C.

the most effective NMES electrode placement for the treatment of post-stroke dysphagia by NMES combined with effortful swallowing training.

\section{METHODS}

31 patients with post-stroke dysphagia were enrolled for this study, which was conducted at the Department of Rehabilitation Medicine, Kyungpook National University Hospital, Daegu, South Korea, between 1 January 2016 and 30 September 2017. The inclusion criteria were: stroke confirmed on computed tomography or magnetic resonance imaging; post-stroke dysphagia confirmed on videofluoroscopic swallowing study (VFSS); and sufficient language and cognitive function to perform effortful swallowing training. The exclusion criteria were: other neurologic diseases and medical condition(s) that represented a contraindication to NMES, such as cardiac pacemaker and epilepsy. This research was approved by the institutional review board of Kyungpook National University Hospital (approval no. KNUMC_15-1021). All patients included in the study provided informed consent prior to participation.

This study was designed as a single-blind, randomised controlled trial with a blinded observer. A computer-generated randomisation sequence and an automated assignment system were used for allocation. 31 patients with post-stroke dysphagia were randomly allocated into the three groups according to NMES electrode placements. In Group A, one pair of electrodes was attached on the suprahyoid muscles horizontally just above both ends of the hyoid bone based on palpation of the hyoid bone. A second pair of electrodes was attached horizontally on the infrahyoid muscles inferior to the hyoid bone for these patients. In Group B, the edge of the hyoid bone was first detected by palpation and one pair of electrodes attached on the suprahyoid muscles horizontally just above both ends of the hyoid bone. The other pair of electrodes was attached vertically on the infrahyoid muscles at the midline inferior to the hyoid bone for these patients. In Group C, all electrodes were attached vertically along the midline after verifying the thyroid notch by palpation. The first electrode was placed across the hyoid bone above the thyroid notch and the second electrode was placed immediately superior to the first. The third electrode was placed inferior to the thyroid cartilage and the fourth electrode was placed directly inferior to the third (Fig. 1). The NMES electrodes were applied by an experienced occupational therapist for all patients.

The electrical stimulation unit (VitalStim ${ }^{\circledR}$; Chattanooga Group, Hixson, TN, USA) used in this study provided a pulse rate of $80 \mathrm{~Hz}$, with biphasic pulse duration of $300 \mu$ s. The amplitude of the electric current (range $0-25 \mu \mathrm{A}$ ) could be adjusted independently for each of the two stimulation channels. The skin in the submental and laryngeal regions was cleaned using a sterile alcohol swab, and two pairs of bipolar surface electrodes were attached. After placing the electrodes on the patient's neck, stimulation intensity for each pair of electrodes was increased in increments of $0.5 \mu \mathrm{A}$ until the patient reported feeling a tingling sensation. Then, the stimulation intensity for each channel was increased until muscle contraction was visible.

The patients performed effortful swallowing training simultaneously with NMES. Specifically, patients were repeatedly asked to forcefully swallow their saliva every ten seconds during stimulation in order to elevate the hyolaryngeal complex, followed by a ten-second rest period. Each intervention session lasted 20 minutes and five sessions were performed weekly for four weeks.

The effect of NMES electrode placement was assessed in terms of the Functional Dysphagia Scale (FDS) and Dysphagia Outcome and Severity Scale (DOSS) scores. The FDS is a 100-point scale evaluating the oral (FDS-O) and pharyngeal (FDS-P) phases of swallowing. The components of FDS-O are lip closure, bolus formation, residue in the oral cavity and oral transit time. The components of FDS-P are triggering of pharyngeal swallow, laryngeal elevation and epiglottic closure, nasal penetration, residue in the valleculae, residue in the pyriform sinuses, coating of the pharyngeal wall after swallowing, and pharyngeal transit time. ${ }^{(18)}$ The DOSS is a simple seven-point scale that evaluates the level of independence, tolerated diet consistency and extent of nutritional restrictions. ${ }^{(19)}$

VFSS was performed to evaluate dysphagia at baseline and after the intervention. While seated, each patient was asked 
Table I. Characteristics of patients with dysphagia undergoing NMES treatment.

\begin{tabular}{|lllll|}
\hline Characteristic & \multicolumn{3}{c}{ No./mean \pm SD } & \multicolumn{2}{c|}{ p-value } \\
\cline { 2 - 4 } & Group A ( $\mathbf{n}=\mathbf{1 0})$ & Group B (n= 11) & Group C (n= 10) \\
\hline Age (yr) & $64.80 \pm 14.05$ & $60.45 \pm 16.15$ & $62.40 \pm 12.65$ & 0.79 \\
\hline Gender (male:female) & $9: 1$ & $8: 3$ & $5: 5$ & 0.15 \\
\hline Stroke lesion (supratentorial:infratentorial) & $8: 2$ & $10: 1$ & $7: 3$ & 0.51 \\
\hline Stroke type (infarction:haemorrhage) & $6: 4$ & $4: 7$ & $4: 6$ & 0.54 \\
\hline Time from onset to initial VFSS (day) & $17.40 \pm 11.52$ & $29.00 \pm 23.71$ & $18.20 \pm 15.82$ & 0.27 \\
\hline
\end{tabular}

$\mathrm{p}<0.05$ is considered statistically significant. NMES: neuromuscular electrical stimulation; SD: standard deviation; VFSS: videofluoroscopic swallowing study

Table II. Within-group comparisons of scores of patients before and after NMES treatment.

\begin{tabular}{|c|c|c|c|c|c|c|c|c|c|}
\hline \multirow[t]{3}{*}{ Variable } & \multicolumn{9}{|c|}{ Score (mean \pm SD) } \\
\hline & \multicolumn{3}{|c|}{ Group A } & \multicolumn{3}{|c|}{ Group B } & \multicolumn{3}{|c|}{ Group C } \\
\hline & $\begin{array}{l}\text { Before } \\
\text { treatment }\end{array}$ & $\begin{array}{l}\text { After } \\
\text { treatment }\end{array}$ & p-value & $\begin{array}{l}\text { Before } \\
\text { treatment }\end{array}$ & $\begin{array}{l}\text { After } \\
\text { treatment }\end{array}$ & p-value & $\begin{array}{l}\text { Before } \\
\text { treatment }\end{array}$ & $\begin{array}{l}\text { After } \\
\text { treatment }\end{array}$ & p-value \\
\hline FDS & $55.02 \pm 16.01$ & $19.40 \pm 8.80$ & $<0.01^{*}$ & $54.91 \pm 14.18$ & $39.82 \pm 12.53$ & $<0.01^{*}$ & $58.70 \pm 14.12$ & $40.70 \pm 18.37$ & $<0.01^{*}$ \\
\hline FDS-O & $12.40 \pm 5.62$ & $8.60 \pm 4.22$ & $<0.01^{*}$ & $16.73 \pm 5.35$ & $12.55 \pm 3.88$ & $<0.01^{*}$ & $18.70 \pm 5.31$ & $14.70 \pm 4.85$ & $<0.01^{*}$ \\
\hline FDS-P & $42.80 \pm 12.08$ & $10.08 \pm 5.59$ & $<0.01^{*}$ & $38.18 \pm 9.78$ & $27.27 \pm 10.17$ & $<0.01^{*}$ & $40.00 \pm 10.07$ & $27.00 \pm 14.61$ & $<0.01^{*}$ \\
\hline DOSS & $2.70 \pm 1.06$ & $6.10 \pm 0.57$ & $<0.01^{*}$ & $3.19 \pm 1.54$ & $4.82 \pm 1.40$ & $<0.01^{*}$ & $3.00 \pm 1.49$ & $4.40 \pm 1.78$ & $<0.01^{*}$ \\
\hline
\end{tabular}

${ }^{*} \mathrm{p}<0.05$ is considered statistically significant within each group. DOSS: Dysphagia Outcome and Severity Scale; FDS: Functional Dysphagia Scale; FDS-O: oral phase of FDS; FDS-P: pharyngeal phase of FDS; NMES: neuromuscular electrical stimulation; SD: standard deviation

to swallow $6 \mathrm{~mL}$ of diluted barium three times. The patients were observed in the lateral and anteroposterior planes. All procedures were recorded in a digital video file and analysed by two physiatrists. The FDS and DOSS scores were established based on the VFSS findings.

All statistical analyses were conducted using IBM SPSS Statistics version 19 for Windows (IBM Corp, Armonk, NY, USA). The Wilcoxon signed-rank test was used for within-group comparisons of the changes in scores following intervention. The Kruskal-Wallis test was used for between-group comparisons of intervention-induced changes. Post-hoc comparisons were made using Bonferroni-corrected Mann-Whitney $U$ tests. Results were considered statistically significant if $p<0.05$ on the Wilcoxon signed-rank test or Kruskal-Wallis test. The significance level of the post-hoc comparison was $0.017(0.05 / 3)$.

\section{RESULTS}

31 patients, who consented to participate, were randomised into three intervention groups: Group A $(n=10)$; Group B $(n=11)$; and Group C $(n=10)$. The general characteristics of patients are summarised in Table I. At baseline, there were no significant differences among the groups regarding age, gender, location of brain lesion, type of stroke and time from stroke onset to baseline VFSS. All patients completed the study. Side effects that have been previously reported to be associated with electrical stimulation therapy for dysphagia include skin burns, pain, cardiovascular problems due to vagus nerve activation and laryngeal spasm. ${ }^{(20,21)}$ However, only transient pain that disappeared immediately after cessation of electrical stimulation was noted in the present study. ${ }^{(20,21)}$

At baseline, there was no significant difference among the groups regarding FDS $(p=0.865)$, FDS-O $(p=0.053)$ or FDS-P $(p=0.589)$. In all three groups, all FDS scores (overall, FDS-O and FDS-P) improved significantly after the intervention $(\mathrm{p}<0.01$; Table II). However, the improvement in FDS and FDS-P scores was significantly better in Group A than in Group B (FDS, $p=0.009$; FDS-P, $p=0.005$ ) and Group C (FDS, $p=0.001$; FDS- $P, p=0.001$ ) (Table III and Fig. 2).

At baseline, no significant difference between the groups was noted in terms of DOSS scores $(p=0.734)$. All three groups had significant improvement in DOSS scores after the intervention $(p<0.01 ;$ Table II). However, there was no significant difference between the groups regarding improvement in DOSS scores (Table III and Fig. 2). The improvement in overall FDS and FDS-P scores was significantly higher in Group A than in Groups B and C (Figs. 2a \& c), but no significant differences among the groups were noted for improvement in FDS-O or DOSS scores (Figs. $2 b \& d$ ).

\section{DISCUSSION}

We investigated the effect of NMES combined with effortful swallowing training for post-stroke dysphagia in three groups of patients with distinct placements of electrodes. Although FDS and DOSS scores showed significant improvement after the intervention for all three electrode placements, our results indicated that horizontal electrode placement on the suprahyoid and infrahyoid muscles (Group A) was more beneficial.

NMES is commonly used in therapy for swallowing problems. Lim et al suggested that conventional therapy applied together with NMES electrode placement corresponding to Group A was a better treatment for patients with post-stroke dysphagia than conventional therapy alone. ${ }^{(11)}$ Ludlow et al reported that NMES electrode placement corresponding to Group A may oppose hyoid elevation during swallowing. ${ }^{(12)} \mathrm{Nam}$ et al and Beom et al found good outcomes for NMES electrode placement corresponding to Group B. ${ }^{(13,14)}$ Carnaby-Mann and Crary reported improvement in 

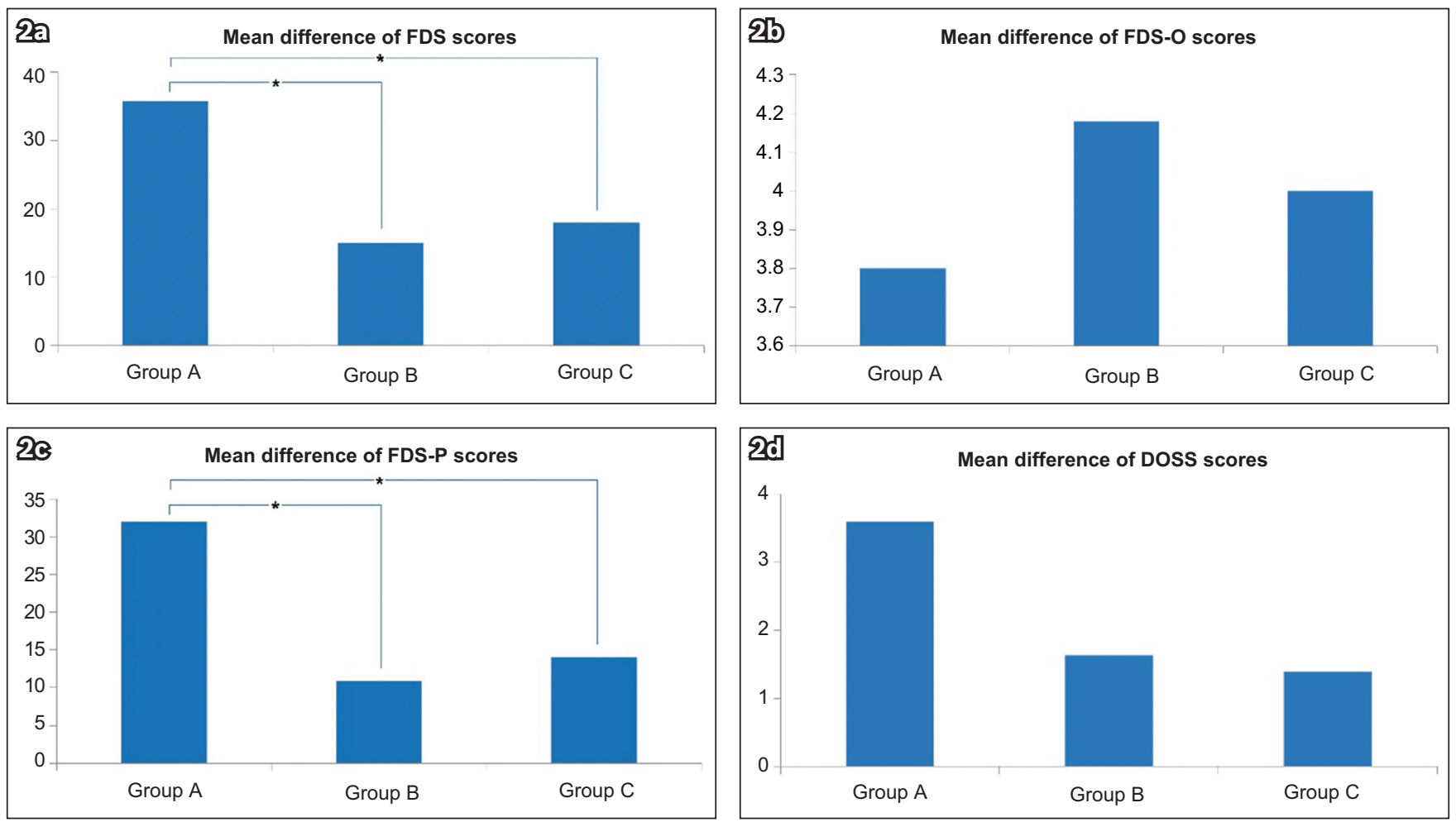

Fig. 2 Charts show the mean differences of (a) FDS scores, (b) FDS-O scores, (c) FDS-P scores and (d) DOSS scores between groups treated under different electrode placements. * $p<0.017$ is considered statistically significant between the groups on post-hoc analysis. DOSS: Dysphagia Outcome and Severity Scale; FDS: Functional Dysphagia Scale; FDS-O: oral phase of FDS; FDS-P: pharyngeal phase of FDS

Table III. Between-group comparisons of scores after NMES treatment.

\begin{tabular}{|c|c|c|c|c|c|c|c|c|c|}
\hline \multirow[t]{2}{*}{ Variable } & \multicolumn{9}{|c|}{ Score (mean \pm SD) } \\
\hline & Group A & Group B & p-value & Group A & Group C & p-value & Group B & Group C & p-value \\
\hline FDS & $35.80 \pm 10.65$ & $15.09 \pm 10.37$ & $0.009^{*, \dagger}$ & $35.80 \pm 10.65$ & $18.00 \pm 12.64$ & $0.001^{*,+}$ & $15.09 \pm 10.37$ & $18.00 \pm 12.64$ & 1.000 \\
\hline FDS-O & $3.80 \pm 2.94$ & $4.18 \pm 2.82$ & $0.019^{*}$ & $3.80 \pm 2.94$ & $4.00 \pm 2.26$ & 0.099 & $4.18 \pm 2.82$ & $4.00 \pm 2.26$ & 0.349 \\
\hline FDS-P & $32.00 \pm 10.83$ & $10.91 \pm 7.97$ & $0.005^{*, \dagger}$ & $32.00 \pm 10.83$ & $14.00 \pm 10.71$ & $0.001^{*,+}$ & $10.91 \pm 7.97$ & $14.00 \pm 10.71$ & 0.863 \\
\hline DOSS & $3.60 \pm 1.26$ & $1.64 \pm 0.67$ & $0.019^{*}$ & $3.60 \pm 1.26$ & $1.40 \pm 1.35$ & $0.029^{*}$ & $1.64 \pm 0.67$ & $1.40 \pm 1.35$ & 0.654 \\
\hline
\end{tabular}

${ }^{*} p<0.05$ is considered statistically significant within each group. $t p<0.017$ was statistically significant between groups on post-hoc analysis. DOSS: Dysphagia Outcome and Severity Scale; FDS: Functional Dysphagia Scale; FDS-O: oral phase of FDS; FDS-P: pharyngeal phase of FDS; NMES: neuromuscular electrical stimulation; SD: standard deviation

clinical swallowing ability and functional oral intake for NMES electrode placement corresponding to Group C. ${ }^{(15)}$ Lim et al reported that NMES electrode placement corresponding to Group $\mathrm{C}$ and low-frequency repetitive transcranial magnetic stimulation treatment were effective for treating patients with dysphagia. ${ }^{(16)}$

In a kinematic analysis of the hyolaryngeal complex during VFSS, Humbert et al reported that the motor effect of stimulation may cause hyolaryngeal lowering. ${ }^{(22)}$ Similarly, Ludlow et al indicated that NMES causes the hyoid bone to move downwards, which may stimulate the patients to make a greater effort to overcome this resistance and swallow. ${ }^{(12)}$ These findings suggest that NMES could be used as a resistance training method, which, when combined with effortful swallowing, may produce a powerful therapeutic effect. Indeed, the kinematic analysis by Park et al demonstrated that effortful swallowing combined with NMES on the sternohyoid muscles lowers the hyoid bone, suggesting that this combined treatment approach may represent a useful rehabilitation strategy. ${ }^{(23)}$
In our study, a four-week intervention programme with horizontal electrode placement above and below the hyoid bone (in Group A) provided significantly better improvement in FDS and FDS-P scores than other placements (in Groups B and C). Humbert et al investigated ten different surface electrode placements covering the submental and laryngeal regions (including the three placements evaluated in our study) on 29 healthy volunteers (age range 20-60 years) without neurological, phonological, psychiatric, speech or swallowing disorders. The study reported the most significant decrease in both hyoid and laryngeal peak elevation for NMES electrode placement corresponding to Group A. ${ }^{(22)}$ Taken together, these findings suggest that horizontal electrode placement above and below the hyoid bone may indeed be the optimal strategy to decrease hyoid and laryngeal peak elevation, which, when combined with effortful swallowing, would produce the most effective rehabilitation via resistance training.

The present study had several limitations. First, the sample size was small and study period short. Thus, our findings may 
not be generalisable to long-term rehabilitation programmes and large patient populations. Future studies with larger sample size and longer study period are necessary. Second, we did not have a control group. Our study was designed more to focus on investigating the most effective electrode placement than the effectiveness of NMES itself. Previous meta-analyses have concluded that NMES tends to improve swallowing function, ${ }^{24,25}$ and therefore we did not include a control group in our study. Lastly, we evaluated dysphagia by using VFSS without kinematic analysis. Additional studies using kinematic analysis of VFSS would be necessary to identify the mechanism that would explain how NMES improves swallowing function.

Despite its limitations, the present study has shown that horizontal placement of electrodes above and below the hyoid bone is more effective than other placements for the treatment of post-stroke dysphagia using NMES therapy combined with effortful swallowing. This rehabilitation strategy could be an effective approach for the treatment of post-stroke patients with dysphagia.

\section{REFERENCES}

1. Katzan IL, Cebul RD, Husak SH, Dawson NV, Baker DW. The effect of pneumonia on mortality among patients hospitalized for acute stroke. Neurology 2003; 60:620-5.

2. Holas MA, DePippo KL, Reding MJ. Aspiration and relative risk of medical complications following stroke. Arch Neurol 1994; 51:1051-3.

3. Horner J, Massey EW. Silent aspiration following stroke. Neurology 1988; 38:317-9.

4. Horner J, Buoyer FG, Alberts MJ, Helms MJ. Dysphagia following brain-stem stroke. Clinical correlates and outcome. Arch Neurol 1991; 48:1170-3.

5. Ashford J, McCabe D, Wheeler-Hegland K, et al. Evidence-based systematic review: oropharyngeal dysphagia behavioral treatments. Part III--impact of dysphagia treatments on populations with neurological disorders. J Rehabil Res Dev 2009; 46:195-204.

6. Singh S, Hamdy S. Dysphagia in stroke patients. Postgrad Med J 2006; 82:383-91

7. Hind JA, Nicosia MA, Roecker EB, Carnes ML, Robbins J. Comparison of effortful and noneffortful swallows in healthy middle-aged and older adults. Arch Phys Med Rehabil 2001; 82:1661-5.
8. Burnett TA, Mann EA, Cornell SA, Ludlow CL. Laryngeal elevation achieved by neuromuscular stimulation at rest. J Appl Physiol (1985) 2003; 94:128-34.

9. Leelamanit V, Limsakul C, Geater A. Synchronized electrical stimulation in treating pharyngeal dysphagia. Laryngoscope 2002; 112:2204-10.

10. Shaw GY, Sechtem PR, Searl J, et al. Transcutaneous neuromuscular electrical stimulation (VitalStim) curative therapy for severe dysphagia: myth or reality? Ann Otol Rhinol Laryngol 2007; 116:36-44.

11. Lim KB, Lee HJ, Lim SS, Choi YI. Neuromuscular electrical and thermal-tactile stimulation for dysphagia caused by stroke: a randomized controlled trial. J Rehabil Med 2009; 41:174-8.

12. Ludlow $\mathrm{CL}$, Humbert I, Saxon K, et al. Effects of surface electrical stimulation both at rest and during swallowing in chronic pharyngeal dysphagia. Dysphagia 2007; 22:1-10.

13. Nam HS, Beom J, Oh BM, Han TR. Kinematic effects of hyolaryngeal electrical stimulation therapy on hyoid excursion and laryngeal elevation. Dysphagia 2013; 28:548-56

14. Beom J, Oh BM, Choi KH, et al. Effect of electrical stimulation of the suprahyoid muscles in brain-injured patients with dysphagia. Dysphagia 2015; 30:423-9.

15. Carnaby-Mann GD, Crary MA. Adjunctive neuromuscular electrical stimulation for treatment-refractory dysphagia. Ann Otol Rhinol Laryngol 2008; 117:279-87.

16. Lim KB, Lee HJ, Yoo J, Kwon YG. Effect of low-frequency rTMS and NMES on subacute unilateral hemispheric stroke with dysphagia. Ann Rehabil Med 2014; 38:592-602.

17. Park JW, Kim Y, Oh JC, Lee HJ. Effortful swallowing training combined with electrical stimulation in post-stroke dysphagia: a randomized controlled study. Dysphagia 2012; 27:521-7.

18. Han TR, Paik NJ, Park JW. Quantifying swallowing function after stroke: a functional dysphagia scale based on videofluoroscopic studies. Arch Phys Med Rehabil 2001; 82:677-82.

19. O'Neil KH, Purdy M, Falk J, Gallo L. The Dysphagia Outcome and Severity Scale. Dysphagia 1999; 14:139-45

20. Freed ML, Freed L, Chatburn RL, Christian M. Electrical stimulation for swallowing disorders caused by stroke. Respir Care 2001; 46:466-74.

21. Huckabee ML, Doeltgen S. Emerging modalities in dysphagia rehabilitation: neuromuscular electrical stimulation. N Z Med J 2007; 120:U2744.

22. Humbert IA, Poletto CJ, Saxon KG, et al. The effect of surface electrical stimulation on hyolaryngeal movement in normal individuals at rest and during swallowing. J Appl Physiol (1985) 2006; 101:1657-63.

23. Park JS, Oh DH, Hwang NK, Lee JH. Effects of neuromuscular electrical stimulation combined with effortful swallowing on post-stroke oropharyngeal dysphagia: a randomised controlled trial. J Oral Rehabil 2016; 43:426-34.

24. Carnaby-Mann GD, Crary MA. Examining the evidence on neuromuscular electrical stimulation for swallowing: a meta-analysis. Arch Otolaryngol Head Neck Surg 2007; 133:564-71.

25. Chen YW, Chang KH, Chen HC, et al. The effects of surface neuromuscular electrical stimulation on post-stroke dysphagia: a systemic review and metaanalysis. Clin Rehabil 2016; 30:24-35. 